\title{
Cisto ósseo traumático: Relato de caso clínico
}

\author{
Traumatic bone cyst: Clinical case report \\ Quiste óseo traumático: Reporte de un caso
}

Gregorio Garcia Lobato Lopez ORCID: https://orcid.org/0000-0002-4614-7396 Hospital Regional Antônio Fontes, Brasil Prefeitura Municipal de Cáceres, Brasil E-mail: gregoriogl12018@gmail.com

Rubens Gonçalves Teixeira

ORCID: https://orcid.org/0000-0003-0814-0940

Faculdade São Leopoldo Mandic, Brasil E-mail: rugonteixeira@gmail.com

\begin{abstract}
Resumo
Cisto ósseo traumático (COT) refere-se a uma lesão rara dos maxilares, denominada por muitos autores como pseudocisto, na maioria das vezes as lesões são descobertas por acaso, em radiografias de rotina. Apresenta-se intraósseo, com cápsula tênue ou tecido conectivo sem epitélio e grande parte das lesões são cavidades vazias, contendo pouco fluido seroso ou serosanguinolento, com cápsula epitelial ausente. O objetivo do estudo foi descrever a clínica de um caso raro de cisto ósseo traumático em uma criança de oito anos, desde seu diagnóstico, até a resolução do caso. Na primeira radiografia panorâmica, feita logo após o trauma, em 07/01/2020 mostra o achado radiográfico de uma lesão, na ocasião não diagnosticada. Pelo trauma houve uma fratura na cortical óssea, ficando associada com essa lesão já existente. Após um ano houve uma reação inflamatória recidivante, expondo o sequestro ósseo, novas imagens, uma anamnese detalhada e principalmente uma busca pela imagem inicial, determinou a existência da lesão ser anterior ao trauma ocorrido. Conforme relato da mãe, houveram outras quedas, anteriores, tornando o trauma a etiologia da lesão. Pela abordagem cirúrgica foi possível o diagnóstico definitivo, o exame anatomopatológico confirmou a hipótese de diagnóstico sendo positivo para COT. O canino inferior esquerdo erupcionando em posição desfavorável foi encaminhado ao serviço de ortodontia. O cirurgião-dentista deve possuir conhecimentos suficientes para diferenciar e diagnosticar as mais diversas lesões císticas, podendo assim proceder rápida e correta resolução dos casos, oferecendo assim um diagnóstico preciso e uma reparação óssea.
\end{abstract}

Palavras-chave: Endodontia; Cisto ósseo traumático; Relatos de casos.

\begin{abstract}
Traumatic bone cyst (TOC) refers to a rare lesion of the jaw, called by many authors as pseudocyst, in most cases the lesions are discovered by chance, in routine radiographs. It presents intraosseous, with a tenuous capsule or connective tissue without epithelium and most of the lesions are empty cavities, containing little serous or serosanguineous fluid, with an absent epithelial capsule. The aim of the study was to describe the clinical features of a rare case of traumatic bone cyst in an eight-year-old child, from diagnosis to case resolution. The first panoramic radiograph, taken right after the trauma, on 01/07/2020 shows the radiographic finding of a lesion, which was not diagnosed at the time. Due to the trauma, there was a fracture in the bone cortical, which was associated with this existing lesion. After one year, there was a recurrent inflammatory reaction, exposing the bone sequestration, new images, a detailed anamnesis, and especially a search for the initial image, determined that the lesion existed prior to the trauma that had occurred. According to the mother's report, there were other, previous falls, making trauma the etiology of the injury. Through the surgical approach, a definitive diagnosis was possible, and the anatomopathological examination confirmed the diagnosis hypothesis being positive for TOC. The lower left canine erupting in an unfavorable position was referred to the orthodontics service. The dentist must have sufficient knowledge to differentiate and diagnose the most diverse cystic lesions, thus being able to proceed quickly and correctly with the resolution of cases, thus offering an accurate diagnosis and bone repair.
\end{abstract}

Keywords: Endodontics; Traumatic bone cyst; Case report.

\section{Resumen}

El quiste óseo traumático (TOC) se refiere a una lesión rara de la mandíbula, denominada por muchos autores como pseudoquiste, en la mayoría de los casos las lesiones se descubren por casualidad, en radiografías de rutina. Se presenta intraósea, con cápsula tenue o tejido conectivo sin epitelio y la mayoría de las lesiones son cavidades vacías, conteniendo poco líquido seroso o serosanguíneo, con ausencia de cápsula epitelial. El objetivo del estudio fue describir las 
características clínicas de un caso raro de quiste óseo traumático en un niño de ocho años, desde el diagnóstico hasta la resolución del caso. La primera radiografía panorámica, tomada inmediatamente después del trauma, el 01/07/2020, muestra el hallazgo radiográfico de una lesión no diagnosticada. Debido al trauma, hubo una fractura en la cortical ósea, que se asoció con esta lesión existente. Luego de un año, hubo una reacción inflamatoria recurrente, exponiendo el secuestro óseo, nuevas imágenes, una anamnesis detallada y sobre todo una búsqueda de la imagen inicial, determinó que la lesión existía antes del trauma ocurrido. Según relato de la madre, hubo otras caídas previas, haciendo del trauma la etiología de la lesión. A través del abordaje quirúrgico fue posible el diagnóstico definitivo y el examen anatomopatológico confirmó que la hipótesis diagnóstica era positiva para COT. El canino inferior izquierdo que erupcionaba en una posición desfavorable fue remitido al servicio de ortodoncia. El odontólogo debe tener los conocimientos suficientes para diferenciar y diagnosticar las más diversas lesiones quísticas, pudiendo así proceder rápida y correctamente con la resolución de los casos, ofreciendo así un diagnóstico certero y reparación ósea.

Palabras clave: Endodoncia; Quiste óseo traumático; Informes de casos.

\section{Introdução}

Cisto ósseo traumático (COT) refere-se a uma lesão rara dos maxilares, denominada por muitos autores como pseudocisto, na maioria das vezes as lesões são descobertas por acaso, em radiografias de rotina. Intraósseo, apresentando cápsula tênue ou tecido conectivo sem epitélio, grande parte das lesões são cavidades vazias, contendo pouco fluido seroso ou serosanguinolento, com cápsula epitelial ausente (Lago et al., 2006).

De acordo com a Organização Mundial da Saúde (OMS), o COT é classificado como uma lesão não neoplásica (Barnes et al., 2005), e que pode ser também denominada como: cisto ósseo simples (COS) cisto ósseo solitário, cisto unicameral, cavidade óssea traumática ou cavidade óssea idiopática. Lesão benigna de conteúdo líquido e expansivo, descritas nos ossos longos desde 1832 e foi relatada pela primeira vez nos ossos maxilares em 1929. Possui etiologia e patogênese incerta, sujeita a controvérsias, sendo a localização mais comum dos COT em 90\%, nos ossos longos enquanto que nos ossos gnáticos a frequência é menor que 10\%, sendo na mandíbula a área mais afetada pela lesão (Martins et al., 2011; Paiva et al., 2011), ocorrência na maioria dos casos em região posterior de mandíbula (Harnet et al., 2008). Tendo maior predominância na região de pré-molares e molares da mandíbula (Copete; Kawamata \& Langlais, 1998). COT no ramo mandibular são considerados raros, e com isso representa dificuldade de diagnóstico e etiologia (Forssell et al., 1988). Nos maxilares é considerada uma lesão quase que exclusiva da mandíbula, acometendo na maioria região posteriores, podendo ainda ser localizado também em região de sínfise. Mais raros ainda na maxila, embora existam estudos com 25\% de casos nessa região (Penarrocha et al., 2001).

Radiograficamente, apresenta-se na grande maioria como uma área radiolúcida, bem delimitada, geralmente com contornos festonados entre as raízes podendo causar expansão nas corticais vestibular e lingual de forma discreta. Raramente produz reabsorções radiculares ou deslocamentos dentários (Kaffe; Littner; Buchner, 1982). O presente trabalho relata o caso clínico de um paciente, sexo masculino, oito anos, acometido por um cisto ósseo traumático.

\section{Metodologia}

O presente artigo trata-se de um estudo descritivo de caráter narrativo e reflexivo (Silva-Souza et al., 2021) e foi aprovado pelo comitê de ética em pesquisa da Faculdade São Leopoldo Mandic sob parecer n 4.726.698.

\section{Relato de Caso Clínico}

Paciente do sexo masculino, oito anos de idade, acompanhado pela mãe, compareceu ao serviço de odontopediatria do Centro de Especialidades Odontológicas (CEO), no município de Cáceres-MT, para atendimento emergencial, apresentando processo inflamatório, edema em região intra-oral entre os dentes 31 e 73 com presença de um sequestro ósseo exposto (Figura 1A), queixando dor. Foi solicitada uma radiografia panorâmica (Figura 1B), na qual observou presença de imagem radiolúcida, com densidade não homogênea, limites definidos e corticalizados, unilocular, dentição mista saudável, solicitaram também uma 
Research, Society and Development, v. 10, n. 8, e16210816952, 2021

(CC BY 4.0) | ISSN 2525-3409 | DOI: http://dx.doi.org/10.33448/rsd-v10i8.16952

tomografia, o paciente foi medicado e encaminhado ao ambulatório de Cirurgia Buco Maxilo Facial (CTBMF) do Hospital Regional de Cáceres (HRCAF) para avaliação e planejamento de tratamento.

Figura 1. Região Intra-Oral com presença de sequestro ósseo exposto (A), radiografia panorâmica (B), tomografia em cortes axiais (C) e cortes transversais (D).

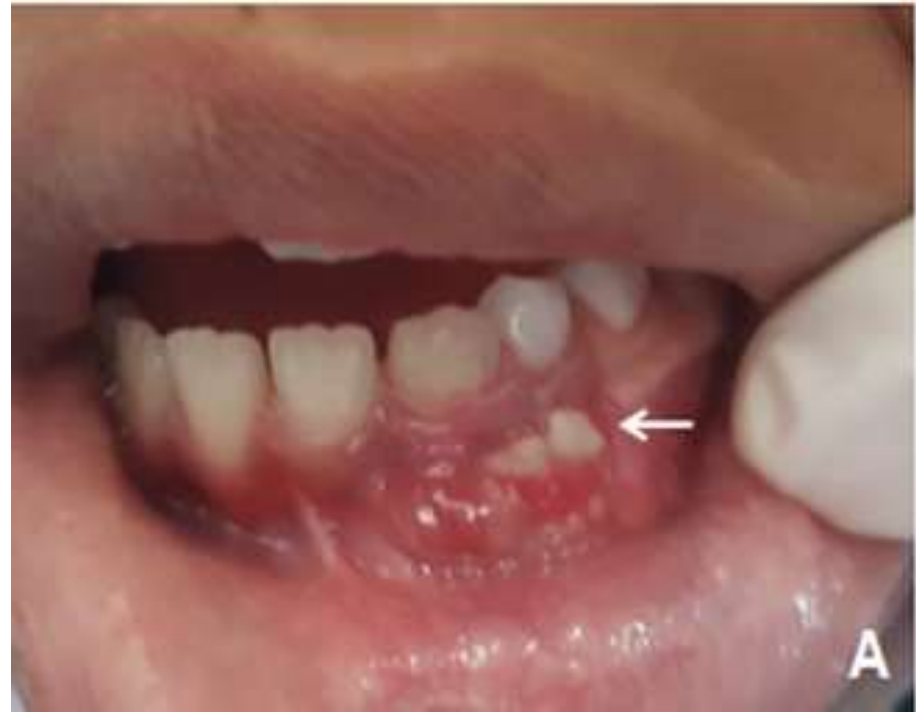

Fonte: Autores(25/11/2020).

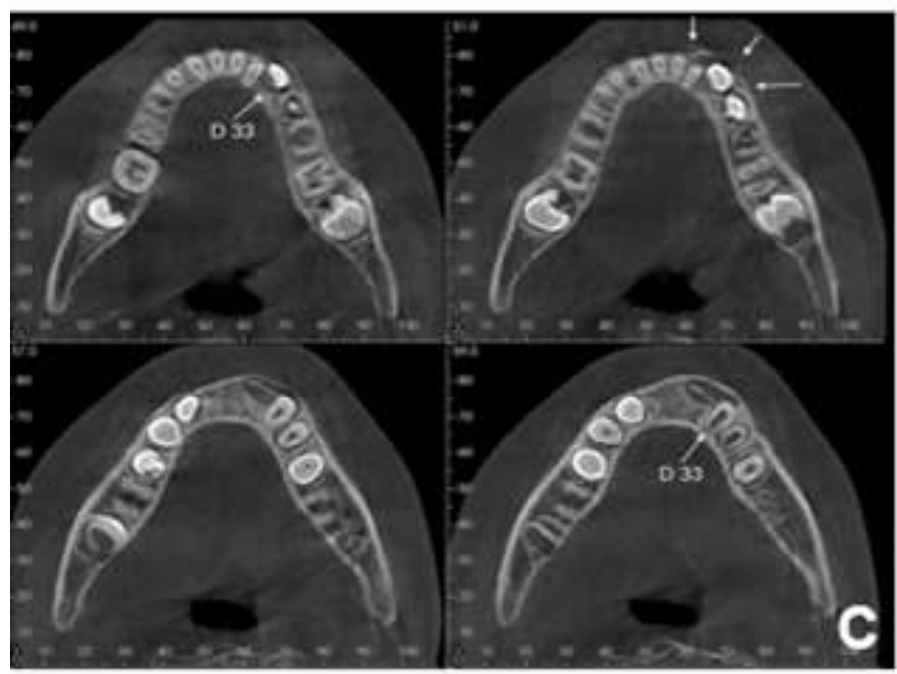

Fonte: Autores (14/12/2020).

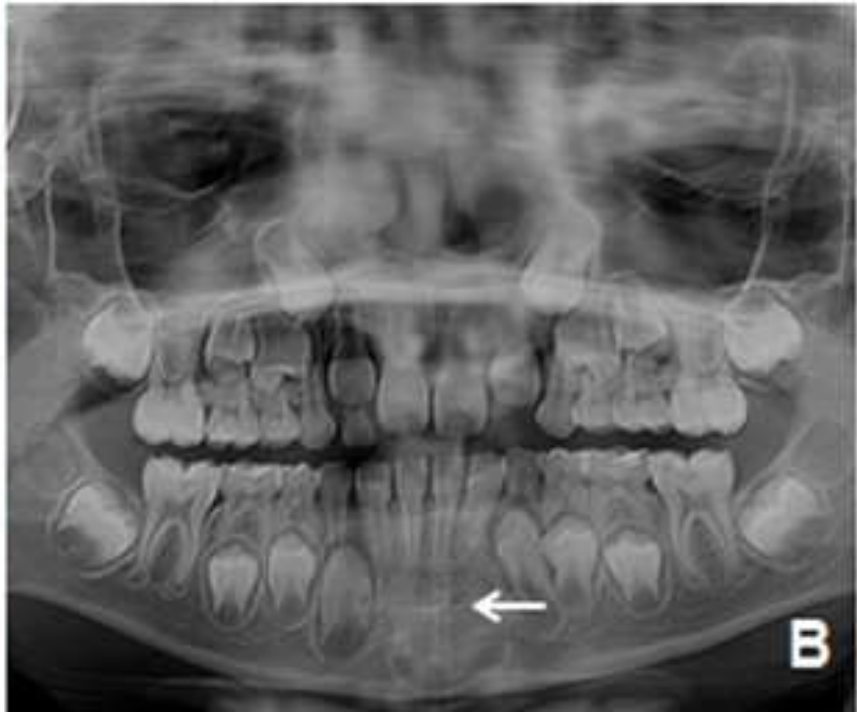

Fonte: Autores (30/11/2020).

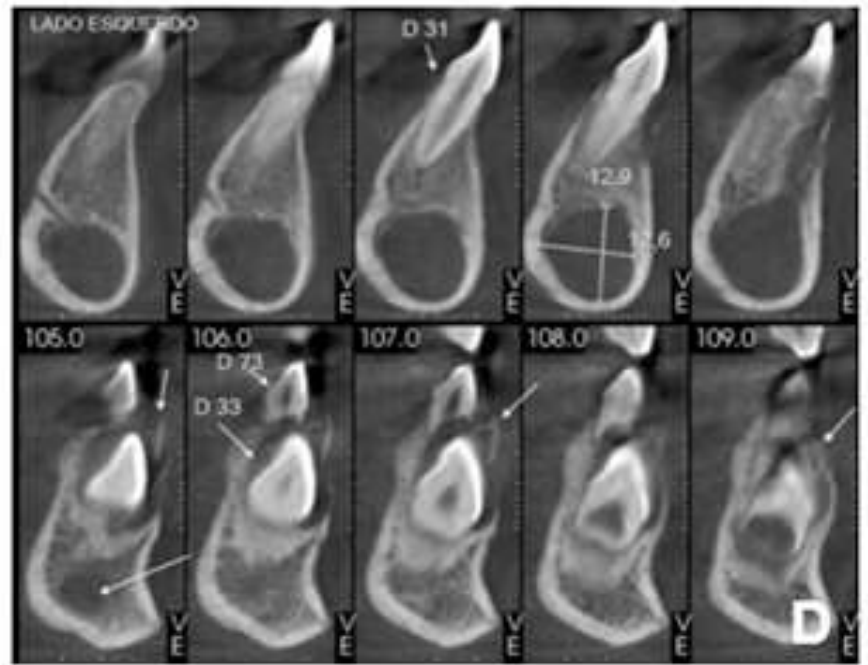

Fonte: Autores (14/12/2020).

\subsection{Ambulatório CTBMF do HRCAF}

No atendimento ambulatorial buco maxilo do HRCAF, na anamnese observou-se histórico de um trauma acidental ocorrido no final de dezembro/2019 (Fig. 2A), na ocasião foi atendido no serviço privado de odontologia, feito uma radiografia panorâmica em 07/01/2020 (Figura 2B) e medicado. Após 30 dias formou-se um edema na região do trauma (Figura 2C), foi abordado com uma drenagem e tratado com antibiótico e anti-inflamatório, não foi diagnosticado fratura de cortical óssea ou 
observado o achado radiográfico da lesão cística já existente e provavelmente por essa razão, não houve acompanhamento. Após um ano do episódio voltou novamente o edema (Fig.1A) e dessa vez atendido pela odontopediatria do CEO e encaminhado ao ambulatório CTBMF do hospital regional. Feito anamnese, avaliadas todas as imagens disponíveis pelas radiografias panorâmicas e tomografias, solicitado exames laboratoriais pré-operatórios, avaliação da cardiologia (risco cirúrgico) e programado retorno para agendamento do tratamento da lesão cística. No retorno, após avaliação dos exames solicitados, foi emitido laudo para regulação na secretaria municipal de saúde e agendado tratamento cirúrgico.

Figura 2. Trauma ocorrido no final de dezembro/2019 (A); radiografia panorâmica realizada em 07/01/2020 (B) já mostrando a lesão sem relação com o episódio traumático. Edema extra oral no final de janeiro/2020 (C).

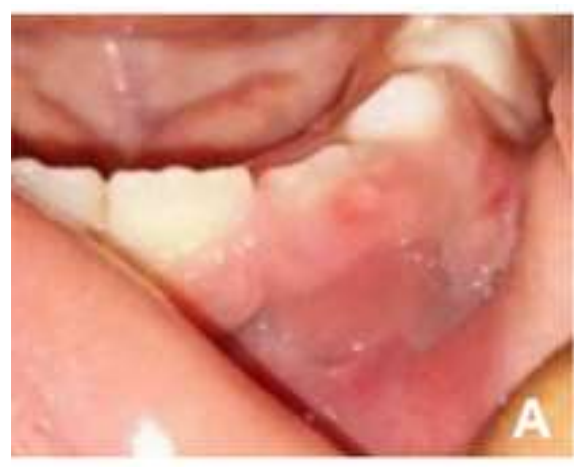

Fonte: Autores (Final de dezembro/2019)

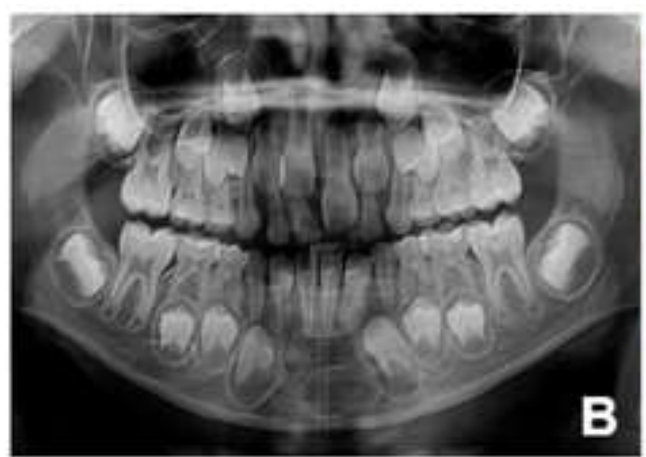

Fonte: Autores (07/01/2020)

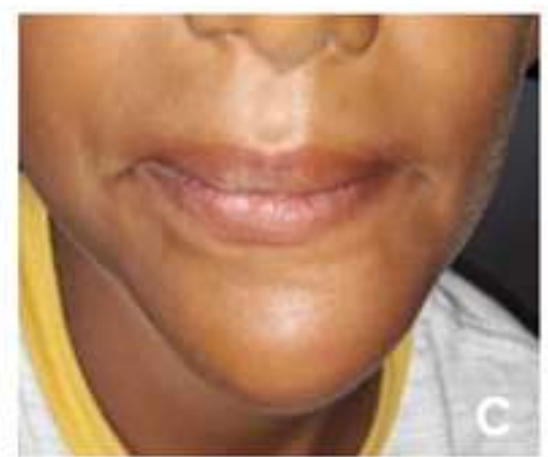

Fonte: Autores (Final de Janeiro/2020).

\subsection{Tratamento Cirúrgico}

Paciente acompanhado pela mãe foi atendido em consulta pelo anestesiologista, reavaliado todos os exames anteriormente solicitados, optou-se por anestesia geral em razão da pouca idade, paciente estar ansioso e não colaborativo. Após manobra anestésica, paciente em posição de decúbito dorsal com intubação oral, feito antissepsia intra e extra-oral, aposição de campos operatórios. Instalou-se o tampão orofaríngeo, infiltrado anestésico local em região de fundo de vestíbulo, na região mentoniana (lidocaína 2\% com epinefrina 1:200.000). Manobra semiotécnica da punção aspirativa antes da exploração cirúrgica (Fig.3A), conteúdo sanguinolento com ausência de cristais de colesterol. Iniciou-se o procedimento cirúrgico com acesso à região com retalho triangular, preservando feixe neurovascular mentoniano esquerdo, osteotomia para acesso da loja cirúrgica com abundante irrigação com SF 0,9\%, removido sequestro ósseo, promovendo assim um acesso cirúrgico na parte superior da lesão (Fig.3B), curetagem na loja e irrigação com SF 0,9\%, sutura com fio reabsorvível, todo material coletado foi conservado no formol 10\% e enviado para exame anatomopatológico (Figura 3C e 3D). Após recuperação anestésica o paciente foi mantido em observação na clínica pediátrica. Alta hospitalar com prescrição de antibiótico, anti-inflamatório, analgésico, antisséptico oral, orientações e solicitado retorno pós-operatório para acompanhamento. 
Figura 3. Procedimento cirúrgico de Punção Aspirativa (A); Remoção Cirúrgica (B); Material coletado que foi encaminhado para exame anatomopatológico (C) e resultado do exame sendo positivo para diagnóstico de COT (D).

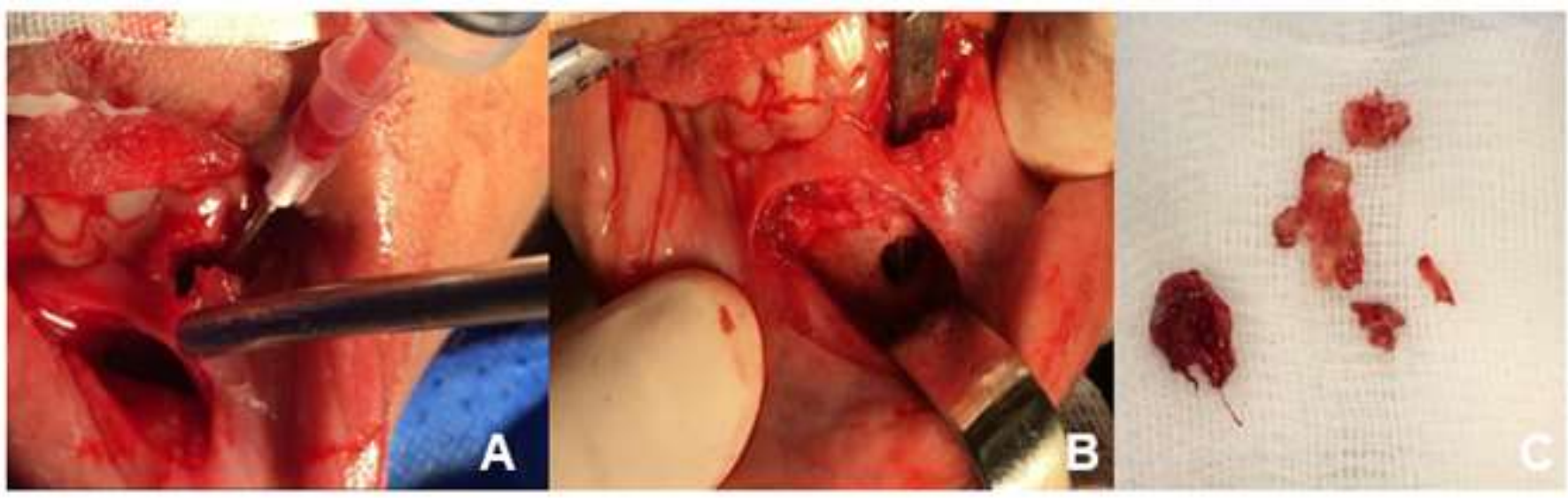

Fonte: Autores $(05 / 02 / 2021)$

Convênio: SUS

Sexo: $M$

Material: REGIĀO ANTERIOR DA MANDIBULA

Idade: $8 a$

Exame: AP21270

Entrada: 08/02/2021

Liberado: $17 / 02 / 2021$

\section{RELATÓRIO MÉDICO DE EXAME ANATOMOPATOLÓGICO}

INFORMAÇÃO CLÍNICA

Lesão em região anterior de mandibula, com características císticas.

HD: Cisto ósseo traumático?

MACROSCOPIA

Recebido em formalina à $10 \%$, fragmentos ósseos e de mucosa oral, irregulares, acastanhados que medem em conjunto $2,0 \times 1,6 \times 0,6$

$\mathrm{cm}$. Todo o material foi submetido a análise histológica. $\mathrm{VF} / 2 \mathrm{C}$

\section{DIAGNÓSTICO}

LESÃO EM REGIÃO ANTERIOR DA MANDÍBULA:

- Amostra composta por fragmentos de mucosa epitelial oral com extensa ulceração associado a tecido de granulação e fragmentos ósseos com alteraçōes reparativas

- Conjunto dos achados podem corresponder a hipotese clínica cisto ósseo traumático

Nota: Não há evidências de malignidade nesta amostra.

Fonte: Autores (17/02/2021) 
Figura 4. Radiografia com 60 dias de pós operatório (A) e imagem fotográfica de 60 dias também de pós operatório (B), paciente foi encaminhado ao serviço de ortodontia para avaliação e conduta para reposicionamento do dente 33.

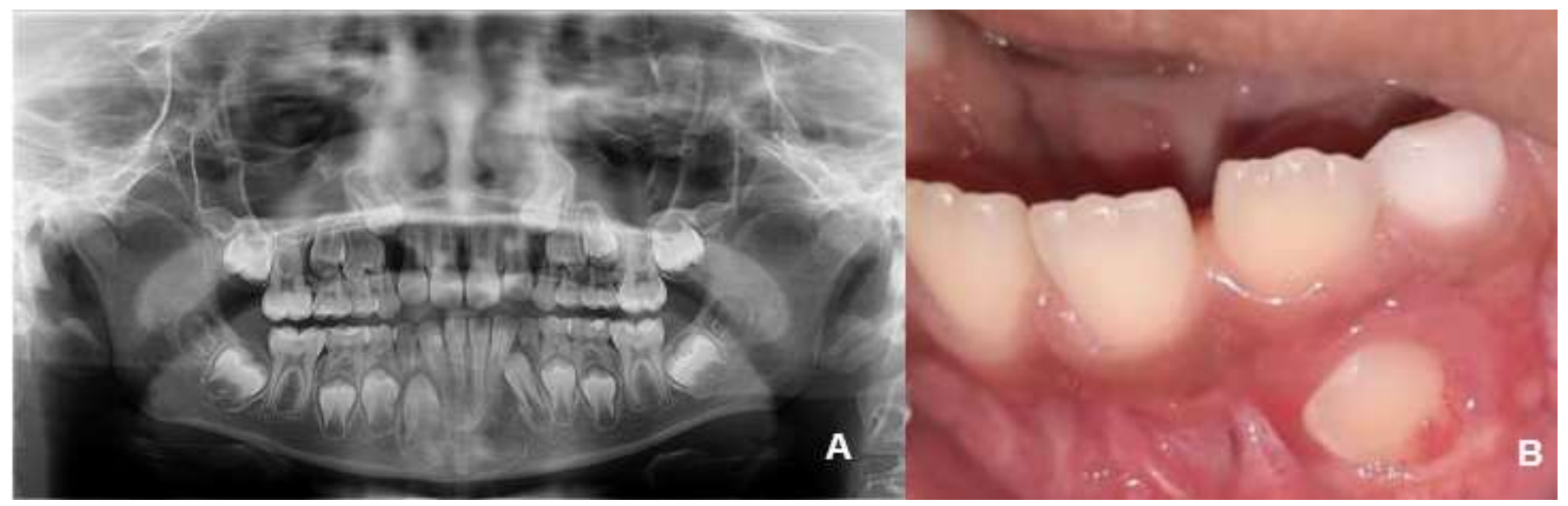

Fonte: Autores (22/04/2021)

\section{Discussão}

O COT é uma patologia frequentemente identificada no osso mandibular e de diagnóstico difícil, devido à semelhança radiográfica com outras lesões (Harnet et al, 2008).

No presente estudo o paciente com oito anos, sexo masculino, como encontrado na literatura, enquadra-se no perfil epidemiológico dos COT. Existem várias hipóteses etiológicas para os casos de COT mandibular, predominando: anormalidade no crescimento ósseo, degeneração tumoral e a teoria mais aceita atualmente, conhecida como traumático-hemorrágico em que a falta de resolução ou lise do coágulo intramedular após o trauma é o responsável pela lesão, isso pode explicar sua maior ocorrência em pacientes jovens, ocorrendo com maior incidência nas primeiras duas décadas de vida (Harnet et al., 2008).

A presença de sintomatologia é rara no COT, e quando ocorrem se dá por meio do aumento volumétrico, parestesia, dor ou fratura patológica. Em exame radiográfico, mostra uma lesão radiolúcida bem definida com morfologia variada. Pode ocorrer expansão óssea, e raramente ocorrem reabsorções dentárias. Histopatológicamente mostra janela cística com membrana de tecido conectivo, sem cápsula epitelial, fibras colágenas, fibroblastos e células gigantes radiculares que podem ser visíveis, nova formação trabecular, capilares congestos e cristais de colesterol, além de necrose óssea (Lago et al., 2005; Jesus et al., 2010).

Válido ressaltar que o diagnóstico, tratamento e resolução dos casos de COT nos maxilares são de competência exclusiva do cirurgião-dentista. Sendo assim indispensável seu conhecimento para diagnóstico e intervenção precoce dos casos (Teixeira et al., 2012).

No presente caso a análise radiográfica inicial quando ocorreu o trauma (Fig.2B) foi determinante para elucidar a presença do COT anterior ao trauma sofrido.

A exploração cirúrgica utilizada no presente caso é o tratamento indicado para COT (Kumar; Kurien \& Thahan, 2015). Sendo primordial para conclusão do diagnóstico, que posteriormente foi confirmado com o laudo histopatológico. Estudos referente ao tema ressaltam a importância da associação de uma boa avaliação do histórico clínico do paciente, exame físico, radiográfico, exploração cirúrgica e resultados dos exames histopatológicos, para uma boa resolução e tratamento de casos de COT (Chapanov; Kazakov \& Iliev, 2020).

O diagnóstico do COT somente pode ser estabelecido após o acesso cirúrgico à cavidade óssea. Seu tratamento é relativamente simples e com bom prognóstico. Segundo alguns autores, é uma lesão que pode ter cura espontânea, sem 
tratamento, entretanto é um tópico controverso. A intervenção cirúrgica geralmente é indicada por oferecer um diagnóstico definitivo e também pelo fato de que uma simples exploração da lesão costuma ser o tratamento adequado, a curetagem das paredes ósseas favorece uma regeneração após alguns meses.

\section{Conclusão}

Na primeira radiografia panorâmica, feita logo após o trauma, em 07/01/2020 mostra o achado radiográfico de uma lesão, na ocasião não diagnosticada. Pelo trauma houve uma fratura na cortical óssea, ficando associada com essa lesão já existente. Após um ano houve uma reação inflamatória redicivante, expondo o sequestro ósseo, novas imagens, uma anamnese detalhada e principalmente uma busca pela imagem inicial, determinou a existência da lesão ser anterior ao trauma ocorrido. Conforme relato da mãe, houveram outras quedas, anteriores, tornando o trauma a etiologia da lesão. Pela abordagem cirúrgica foi possível o diagnóstico definitivo, o exame anatomopatológico confirmou a hipótese de diagnóstico sendo positivo para COT. O canino inferior esquerdo erupcionando em posição desfavorável foi encaminhado ao serviço de ortodontia.

O cirurgião-dentista deve possuir conhecimentos suficientes para diferenciar e diagnosticar as mais diversas lesões císticas, podendo assim proceder rápida e correta resolução dos casos, oferecendo assim um diagnóstico preciso e uma reparação óssea.

\section{Referências}

Barnes, L., Eveson, J., Reichart, P., \& Sidransky, D. (2005). World Health Organization classification of tumours: pathology and genetics of head and neck tumours. World Health Organization Classification of Tumors: blue book.

Harnet, J. C., Lombardi, T., Klewansky, P., Rieger, J., Tempe, M. H., \& Clavert, J. M. (2008). Solitary bone cyst of the jaws: a review of the etiopathogenic hypotheses. Journal of Oral and Maxillofacial Surgery, 66(11), 2345-2348.

Isola, G., Cicciù, M., Fiorillo, L., \& Matarese, G. (2017). Association between odontoma and impacted teeth. Journal of Craniofacial Surgery, 28(3), 755-758.

Kumar, L. S., Kurien, N., \& Thaha, K. A. (2015). Traumatic bone cyst of mandible. Journal of maxillofacial and oral surgery, 14(2), 466-469.

Chapanov, K.; Kazakov, S.; Iliev, G. (2020) Traumatic bone cyst of the mandible: A Case Report. MedInform, 2.

Lago, C. A., Cauás, M., Pereira, A. M., \& Portela, L. (2006). Cisto ósseo traumático em mandíbula: relato de caso. Rev Cir Traumatol Buco-Maxilo-Fac, 6(2), $17-22$.

Jesus, V. A. D. D., Santos, T. D. S., Fernandes, A. V., \& Santos, J. S. (2010). Cisto ósseo traumático: relato de caso. Revista de Cirurgia e Traumatologia Bucomaxilo-facial, 10(4), 027-030.

Martins-Filho, P. R. S., Santos, T. D. S., Araújo, V. L. C. D., Santos, J. S., Andrade, E. S. D. S., \& Silva, L. C. F. D. (2012). Cisto ósseo traumático da mandíbula: revisão de 26 casos. Brazilian Journal of Otorhinolaryngology, 78(2), 16-21.

Paiva, L. C. D. A., Menezes, F. D. S., Porto, G. G., \& Cerqueira, P. R. F. (2011). Cisto Ósseo Simples-Relato de Caso. Revista de Cirurgia e Traumatologia Buco-maxilo-facial, 11(2), 15-20.

Razmara, F., Ghoncheh, Z., \& Shabankare, G. (2019). Traumatic bone cyst of mandible: a case series. Journal of medical case reports, 13(1), 1-8.

Silva, M. C., de Lima Neto, T. J., de Souza Santos, A. M., Faverani, L. P., Inaoka, S. D., \& da Costa, D. F. N. (2021). Cisto ósseo traumático em área incomum: relato de caso. Archives of health investigation, 10(1), 170-173.

Teixeira, R. G.; et al. (2012). Cisto Ósseo Simples: Relato de caso clínico. ACDC em ação, 1(153).

Dellinger, T. M., Holder, R., Livingston, H. M., \& Hill, W. J. (1998). Alternative treatments for a traumatic bone cyst: a longitudinal case report. Quintessence international, 29(8).

Marzola, C. (2005). Fundamentos de cirurgia e traumatologia buco-maxilo-facial. Bauru: Independente.

Jesus, V. A. D. D., Santos, T. D. S., Fernandes, A. V., \& Santos, J. S. (2010). Cisto ósseo traumático: relato de caso. Revista de Cirurgia e Traumatologia Bucomaxilo-facial, 10(4), 027-030.

Lago, C. A., Cauás, M., Pereira, A. M., \& Portela, L. (2006). Cisto ósseo traumático em mandíbula: relato de caso. Rev Cir Traumatol Buco-Maxilo-Fac, 6(2), 17-22. 
Research, Society and Development, v. 10, n. 8, e16210816952, 2021

(CC BY 4.0) | ISSN 2525-3409 | DOI: http://dx.doi.org/10.33448/rsd-v10i8.16952

Lokiec, F., \& Wientroub, S. (1998). Simple bone cyst: etiology, classification, pathology, and treatment modalities. Journal of pediatric orthopedics. Part B, $7(4), 262-273$.

Shear, M. (1999). Cistos da região bucomaxilofacial: diagnóstico e tratamento. Santos.

Shimoyama, T., Horie, N., Nasu, D., Kaneko, T., Kato, T., Tojo, T., ... \& Ide, F. (1999). So-called simple bone cyst of the jaw: a family of pseudocysts of diverse nature and etiology. Journal of oral science, 41(2), 93-98.

Shafer, W. G.; Hine, M. K.; Levy, B. M. (1985). Tratado de patologia bucal.

Silva-Souza, L. G., de Oliveira, L. D., Nunes, G. P., dos Santos Cividanes, L., Dahan, C. M., Pereira, A. K. G., ... \& e Silva, L. F. D. C. (2021). Effectiveness of the low-level laser therapy in the management of bisphosphonate-induced osteonecrosis of the jaws: A case report. Research, Society and Development, 10(6), e17510615199-e17510615199.

Takahama Junior, A., Pires, F. R., Jorge, J., \& Lopes, M. A. (2007). Cisto ósseo simples: relato de quatro casos e revisão de literatura. Rev. Assoc. Paul. Cir. Dent, 488-491.

Telfer, M. R., Jones, G. M., Pell, G. M., \& Eveson, J. W. (1990). Primary bone cyst of the mandibular condyle. British Journal of Oral and Maxillofacial Surgery, 28(5), 340-343.

Whinery, J. G. (1955). Progressive bone cavities of the mandible: a review of the so-called traumatic bone cyst and a report of three cases. Oral Surgery, Oral Medicine, Oral Pathology and Oral Radiology, 8(9), 903-916.

Winer, R. A., \& Doku, H. C. (1978). Traumatic bone cyst in the maxilla. Oral Surgery, Oral Medicine, Oral Pathology, 46(3), 367-370.

Wood, N. K., \& Goaz, P. W. (1997). Differential diagnosis of oral and maxillofacial lesions. Mosby.

Xanthinaki, A. A., Choupis, K. I., Tosios, K., Pagkalos, V. A., \& Papanikolaou, S. I. (2006). Traumatic bone cyst of the mandible of possible iatrogenic origin: a case report and brief review of the literature. Head \& Face Medicine, 2(1), 1-5.

Zegarelli, E. V., Kutscher, A. H., \& Tenore, R. A. (1966). Idiopathic bone cyst. New York state journal of medicine, 66(13), 1753-1756. 